\title{
Topical Corticosteroid
}

National Cancer Institute

\section{Source}

National Cancer Institute. Topical Corticosteroid. NCI Thesaurus. Code C29505.

Any synthetic steroid derivative exhibiting the same function as the naturally occurring corticosteroid hormone, formulated for topical application. Topical corticosteroids are applied to the skin where it exerts its effect, however, corticosteroids can be absorbed systemically after being applied locally. Topical corticosteroids are mainly used for the localized treatment of inflammation of the skin and help relieve symptoms such as itching, swelling and redness. 